\title{
UNED: REVALORACIÓN NECESARIA FRENTE A CAMBIOS HISTÓRICOS Y A TRES CARACTERÍSTICAS SOCIODEMOGRÁFICAS DE SUS GRADUACIONES
}

\author{
MBA. HELLEN RUIZ HIDALGO \\ Universidad Estatal a Distancia, Costa Rica \\ hruiz@uned.ac.cr
}

\section{RESUMEN}

Este artículo expone las transformaciones históricas, en el mundo y en Costa Rica, desde 1977, fecha de creación de la Universidad Estatal a Distancia (UNED), para acentuar cómo sus objetivos fundacionales adquieren mayor relevancia en las condiciones actuales; es decir, en un contexto en el que se han acentuado las brechas sociales, acrecentado la trascendencia de poseer estudios universitarios y revolucionado las tecnologías propias de su modalidad educativa. Existe un desfase entre estos procesos y el peso estratégico de la UNED, que permanece inalterado desde su fundación. El exitoso pero inacabado modelo de internacionalización del país, inmerso como está, en la globalización ha ocasionado también pérdida de cohesión social. Con el análisis de los perfiles de educación secundaria, procedencia socio-geográfica y género de sus graduados, se enfatiza el rol de la UNED como política pública educativa de convergencia entre los contrastes socioeconómicos existentes. Se muestra así la pertinencia de la UNED y se aboga por la revalorización de su rol estratégico.

PALABRAS CLAVE: UNED, EDUCACIÓN, EDUCACIÓN A DISTANCIA, COHESIÓN SOCIAL, POLITICAS DE CONVERGENCIA, MODELO DE DESARROLLO.

\section{ABSTRACT}

This article portrays the historical transformations in the world and in Costa Rica, since 1977, date of creation of the UNED, to emphasize how its foundational objectives acquire greater relevance in the current conditions, when social gaps have been accentuated, the transcendence of having university studies and revolutionizing the technologies proper to its educational modality have been revolutionized. There is a gap between these processes and the strategic weight of the UNED, which has remained unchanged since its foundation. Costa Rica's successful but unfinished model of internationalization, immersed as it is in globalization, has also caused a loss of social cohesion. With the analysis of the profiles of secondary education, socio-geographic origin and gender of its graduates, the role of the UNED is emphasized as a public educational policy ofconvergence between the existing socioeconomic contrasts. This shows the relevance of the UNED and advocates the revaluation of its strategic role.

KEY WORDS: UNED, EDUCATION, DISTANCE EDUCATION, SOCIAL COHESION, CONVERGENCE POLICIES, DEVELOPMENT MODEL. 


\section{INTRODUCCIÓN}

Este artículo presenta un contraste entre las transformaciones socioeconómicas y tecnológicas que han ocurrido, en el mundo y en Costa Rica, desde 1977, fecha de fundación de la UNED, y el rol estratégico inmutable de esta, desde su creación. Desde las postrimerías de los años 70 del siglo pasado, Costa Rica asumió un giro en su modelo de desarrollo, abriéndose unilateralmente al mundo con un proceso de internacionalización reputadamente exitoso. Este cambio, sin embargo, también tuvo como consecuencia un agravamiento de las brechas sociales, territoriales, educativas y asociadas al género. Eso ha creado condiciones de mayores dificultades de acceso a la educación superior, lo que, en sí mismo, refuerza la pertinencia del objetivo fundacional de la UNED, concebida con una modalidad educativa especialmente diseñada para facilitar el ingreso universitario a los segmentos con mayores obstáculos de acceso.

Se discute que de dichos cambios históricos se podría plantear una revalorización del rol estratégico de la UNED. Tanto más en la Sociedad del Conocimiento, cuando el acceso a la educación superior se ha transformado en un elemento que ahonda las brechas sociales entre quienes tienen acceso a formación universitaria y los que quedan excluidos de ella. La educación terciaria es factor decisivo de movilidad social.

Si bien es cierto, la pertinencia de facilitar el acceso a la educación superior por medio de la modalidad educativa a distancia se ha acentuado con el ahondamiento de las brechas y con el creciente rol nivelador de la educación, la función propia de una institución debe ser demostrada más allá de su diseño y de sus intenciones. Por ello, es preciso estudiar y contrastar la relevancia de sus resultados, con los objetivos que persigue según el rol social para el que está diseñada.

Con este propósito, se analizan las graduaciones de la UNED como resultado educativo sociodemográfico. Para ello, se abordan los principales obstáculos de acceso a la educación superior, tales como la deserción en secundaria o baja calidad en este nivel educativo, la procedencia de zonas de bajo índice de desarrollo relativo o de zonas alejadas y la forma en que dichas dificultades se ven agravadas con el factor de género. De ahí que se estudiaran tres características sociodemográficas de las graduaciones de la UNED:

- Graduados que provienen de estudios de secundaria a distancia.

- Graduados de zonas de índice de desarrollo social (IDS) bajo y muy bajo.

- Mujeres graduadas y sus características sociodemográficas.

Con el análisis de esos tres aspectos de los perfiles sociodemográficos de las graduaciones de la UNED, se mostrará la pertinencia de esta universidad para segmentos de población con especiales dificultades de acceso a la educación superior. Esa es otra justificación de la necesidad de revalorización del rol estratégico de la UNED.

En el mundo han tenido lugar transformaciones socioeconómicas y tecnológicas, a partir de la globalización, la revolución de las tecnologías de la información y la comunicación (TIC) y el surgimiento de la Sociedad del Conocimiento. Costa Rica se ha vinculado con esos procesos a partir de su exitoso, pero inacabado, modelo de desarrollo, centrado en una eficiente internacionalización que, al mismo tiempo, ha visto acentuar brechas en todos los órdenes de la vida, con consiguiente pérdida de cohesión social. Estas transformaciones hicieron más trascendental la labor de la UNED, diseñada para responder a brechas y exclusiones que ahora son más perentorias y pusieron a su alcance un instrumental tecnológico 
de mucha mayor eficiencia que el que existía cuando nació.

De lo anterior, podría deducirse que también la prioridad de la UNED, como política pública, se debería haber actualizado, como pareciera corresponder. Es decir, en acordanza con el mayor peso de su necesidad y de la creciente relevancia de los medios tecnológicos para cumplir sus propósitos. A partir de esas circunstancias, se podrían plantear cambios en la proporción en la que participa del financiamiento estatal, en relación con el de otras universidades.

Esto no ha ocurrido así. El peso relativo del presupuesto que se le asigna a la UNED ha permanecido inalterado, con un 9\% del Fondo Especial para el Financiamiento de la Educación Superior (FEES), que termina siendo un $7.51 \%$ que se destina a la función institucional. Eso denota que la visión del Estado sobre su peso estratégico no ha variado desde su fundación.

No es el único tema en el que Costa Rica se encuentra desacoplada frente a los desajustes internos que provocó su desarrollo socioeconómico reciente. Se ha internacionalizado en su estrategia hacia afuera, pero no lo ha hecho de forma integral, con transformaciones estructurales hacia adentro (Govaere, 2018a). En los procesos educativos, asimiló solo parcialmente las tecnologías de la información, con brechas que subsisten en la incorporación de su población a la Sociedad del Conocimiento.

Estos desfases señalan la necesidad de una actualización de políticas complementarias para alcanzar un modelo de desarrollo más acabado, completo en su diseño, sistémico en sus alcances y socioeconómicamente integrado, para que se facilite la convergencia de sectores divididos por diferentes tipos de brechas (Beverinotti, Coj-Sam, y Solís, 2015; Ocampo, 2002).

Se ha señalado que un mejoramiento de la política educativa es una de las adecuaciones que necesita el modelo de desarrollo de Costa Rica. La exposición de algunos matices de los perfiles sociodemográficos de las graduaciones de la UNED 2013-2016 destaca su rol de nivelación social para sectores excluidos o que están en particular desventaja. Eso servirá para subrayar aspectos de eficiencia de la modalidad educativa que la UNED ofrece, como política complementaria de ajuste del modelo. Sin embargo, hasta ahora ha sido escasa la discusión de un replanteamiento estratégico de la UNED.

Esta investigación secundaria, de carácter cualitativo, con referentes bibliográficos y estadísticos, demostrará que los perfiles sociodemográficos de las graduaciones de la UNED tienen capacidad ilustrativa para validar la modalidad educativa a distancia de la UNED como una política pública que ameritaría estudios más amplios de revalorización, dentro de una estrategia-país, más holística en la necesidad de restaurar la cohesión social perdida.

\section{PLANTEAMIENTO DEL PROBLEMA}

En 1977, el Estado de Costa Rica fundó la UNED, como una universidad cuya modalidad educativa "a distancia" la orientó, específicamente, a facilitar el acceso a la educación terciaria, en beneficio de segmentos de población cuya situación social, geográfica o de género les dificultaba el ingreso. Ese era el determinante estratégico del rol social de la UNED, en una Costa Rica que, en esos tiempos, tenía mayor cohesión social, baja desigualdad de ingresos, brechas sociales menos agudas y contrastes territoriales de menor impacto. Ese es el punto de partida para el examen del rol social de la UNED, en la situación actual.

Tomando como ejes de análisis, en primer lugar, literatura académica de referencia y, en segundo lugar, publicaciones estadísticas de algunas características sociodemográficas de las graduaciones de la UNED, se intenta dar un inicio de respuesta a dos interrogantes centrales: 
1. ¿Se mantienen inalterables o, más bien, se han agudizado, entre 1977 y 2019, las condiciones sociales de exclusión a la educación superior que sirvieron de soporte estratégico para la creación de la UNED? La respuesta a esta pregunta concierne a la pertinencia educativa vinculada con el agravamiento de las condiciones que dan origen a su modalidad.

2. ¿Se puede inferir de las características sociodemográficas de las graduaciones de la UNED que su modalidad educativa responde a sus objetivos estratégicos fundacionales? La respuesta a esta pregunta concierne al grado de pertinencia educativa vinculada con los impactos propios a su objetivo fundacional.

La primera pregunta sirve de hipótesis de base. Para responderla, se realiza una amplia investigación de abundantes referencias bibliográficas de soporte en la literatura académica. La segunda pregunta sirve de tesis central de este estudio $y$, para responderla, se realiza una investigación de diferentes fuentes estadísticas del Centro Investigación de Estadísticas Institucionales de la UNED y análisis, en particular de Informes del Estado de la Educación del Programa del Estado de la Nación.

A partir de esas estadísticas, de las que se extraen algunos perfiles historiográficos, se estudian tres perfiles sociodemográficos de las graduaciones 2013-2016 de la UNED, para valorar su impacto estratégico en sectores con desaventajados perfiles educativos, sociales, territoriales y de género. Este estudio busca responder de forma más precisa las siguientes preguntas:

- ¿responde la UNED su objetivo fundacional?

- iresaltan las características sociodemográficas expuestas de esas graduaciones el impacto potencial de la UNED en el mejoramiento de la cohesión social?
- itiene particular pertinencia la UNED como política pública de convergencia?

\section{AGRAVAMIENTO DE CONTRASTES SOCIALES EN COSTA RICA Y EL MUNDO}

Cada vez aparecen más estudios (Jaurata, 1998; Ocampo y Martín, 2003; Serrano, 2005) que muestran que los procesos de inserción en el comercio mundial, a la par de buscados incrementos de riqueza, también conllevan secuelas negativas sistémicas, produciendo brechas en sectores productivos, sociales y territoriales (Stiglitz, 2010; 2015).

Por una parte, están las ventajas que depara la globalización para sectores y territorios directamente vinculados con los procesos de internacionalización y, por otra, los abandonos a los que se ven sometidos sectores y territorios excluidos de sus ventajas o que caen, directa o indirectamente, bajo el peso de una menor competitividad. En esas disparidades aparecen escenarios de "ganadores" y "perdedores", donde se ve menoscabada la cohesión social. Ottone y Sojo (2007) contrastan "aquel... anhelo de comunidad ante un escenario de globalización y transformaciones profundas, que muchos asocian con una mayor fragmentación social" (p. 11).

Cuando no han sido atendidos a tiempo, los contrastes socioeconómicos derivados de la globalización han tenido efectos desestabilizantes. Así se muestra en algunos acontecimientos políticos recientes que se relacionan con formas imperfectas de adecuación de los procesos de globalización a las condiciones de cada país. Desde hace más de diez años, el Estado de la Nación ha venido analizando esta situación en Costa Rica.

...nuevos sectores productivos, que conforman lo que podría denominarse la "nueva economía", coexisten con los principales actores de la "vieja economía"... Así, el país tiene una economía fragmentada: 
importantes sectores de actividad son dinámicos y tecnológicamente avanzados; otros tienen escasa vitalidad y notables rezagos, y son además los que concentran a la mayoría de la población... (Programa Estado de la Nación, 2008, p. 321).

Aludiendo a los resultados de la primera ronda de las elecciones nacionales, de 2018 en Costa Rica, en un artículo de opinión, Govaere (2018b) afirma:

Dos Costa Ricas se enfrentan con dos discursos y dos audiencias. El discurso culto, para un auditorio refinado, se estrella contra el desapego de la empresa informal, el empleo precario, la periferia excluida, la fallida instrucción técnica y la educación sin pertinencia laboral, cuando no truncada. La polarización electoral es el reflejo de esa otra polarización: la social, la productiva, la educativa y la territorial. Esos continentes no calzan en un modelo de desarrollo con la vista puesta solamente en exportaciones. (Govaere, 2018b).

No se ahondará, en este artículo, en la profundización de exclusión social en Costa Rica, ya que esa temática está académicamente muy estudiada. Se alude acá solo a referencias que respaldan ese criterio, tales como: dualidad productiva (Beverinotti et al., 2015; Ocampo, 2002) y brechas sociales vinculadas con la globalización (Govaere, 2018b; Roubini, 2016). Se ha hecho referencia a los dualismos nacidos de diferentes niveles de heterogeneidad característicos de la "modernidad inconclusa" de Costa Rica. Paralelamente, se presenta en estos estudios la necesidad de políticas públicas de contrapeso, referidas con frecuencia como políticas de convergencia, para alcanzar un modelo de desarrollo más acabado. Se consignan los más relevantes: la CEPAL, 2011; por la OIT, Alonso, 2016 y Salazar-Xirinach, 2014; por el BID, Beverinotti, 2016; el Banco Mundial, 2013 y, por la OCDE, Hausman et al., 1966.

\section{INTRODUCCIÓN DEL CONCEPTO DE POLÍTICAS PÚBLICAS DE CONVERGENCIA}

Con lo afirmado anteriormente, no se busca censurar, en sí misma, la apertura comercial ni el modelo de desarrollo que la ubica en un lugar central de las políticas públicas. Se constata, más bien, que los procesos nacionales de internacionalización necesitan de políticas públicas complementarias de acompañamiento para ejercer un contrapeso a los resultados socioeconómicos y regionales que polarizan.

Es una idea generalmente aceptada (Ocampo y Martín, 2003) que cuando los procesos de internacionalización no son acompañados de políticas de contrapeso, que promuevan convergencia de oportunidades, se producen fisuras del tejido social. Estas grietas se manifiestan en divergencias políticas agudas y tienen como resultado sistémico la acentuación y endurecimiento de dualidades nacionales: desigualdad de ingresos, territorios de mayor o menor desarrollo, concentración de la riqueza frente a estancamiento de los segmentos sociales de baja competitividad e ingreso, diferencias de empleabilidad y de competitividad, dualidades productivas, etc. (Programa Estado de la Nación, 2008).

El concepto de "políticas de convergencia" se refiere a acciones estratégicas de Estado que contrarresten la heterogeneidad del dualismo generado con la incorporación imperfecta a la globalización. Se les llama "de convergencia" porque tienen como objetivo disminuir la polarización, reduciendo la desigualdad de ingresos, las brechas territoriales, las diferencias de oportunidades, de productividad y de capital humano.

Dentro de las diversas perspectivas se han ido acumulando muchas propuestas y análisis, que conforman ya un acumulado de visiones que podrían servir de respaldo teórico para un cambio de políticas públicas, centradas fundamentalmente en la convergencia... Estas visiones de ninguna manera 
son "rupturistas" con las políticas públicas de comercio exterior, sino complementarias a ellas. Se tiene conciencia y se defienden los éxitos alcanzados, pero también se proponen políticas públicas de convergencia... con planteamientos holísticos que perfeccionen el modelo. (Govaere, 2018a, p. 608; énfasis de la autora).

\section{LA EDUCACIÓN COMO POLÍTICA PÚBLICA DE CONVERGENCIA}

Ottone y Sojo (2007) consideran que la educación forma parte decisiva de cualquier aproximación integral a la restauración de la cohesión social; es decir, en palabras de Govaere (2018a) y la CEPAL (2011), "políticas de convergencia". La educación es el factor más decisivo de enriquecimiento del acervo de capital humano. Las desigualdades de ingreso, las brechas sociales y los contrastes territoriales al mismo tiempo se derivan de fuertes disparidades educativas y siempre las reproducen. La revolución tecnológica acentúa aún más el rol de la educación en la Sociedad del Conocimiento, pero también crea nuevas brechas.

Existe consenso entre los autores de estimar la educación como política pública de promoción de convergencia estructural porque las brechas educativas acompañan siempre y son componentes importantes de todas las formas de desigualdad. "Distintos estudios han mostrado que la educación es la principal causa del aumento de la desigualdad en Costa Rica" (Trejos, 2015 , p. 2). Con el surgimiento de la Sociedad del Conocimiento, ha estado aumentando el premio salarial para los trabajadores más educados.

(...) la educación surge como requisito tanto para que las personas puedan acceder a los beneficios del progreso, como para que las economías estén en condiciones de garantizar un desarrollo sostenido, gracias a la competitividad basada en el acceso a mayores niveles de educación y capacitación (Arias y Muñoz, 2007, p. 35).

\section{EL MODELO INCONCLUSO DE LA POLÍTICA EDUCATIVA DE COSTA RICA}

La expresión "más maestros que soldados" traduce el valor nacional que tiene la educación dentro de su imaginario colectivo, como factor decisivo de movilidad social (Pérez, 2004). Eso le asigna un rol de convergencia en un entorno de heterogeneidad.

En la primera mitad del siglo XX, los índices de educación de Costa Rica justificaban que la educación fuera parte emblemática de la autoestima colectiva. Costa Rica representaba, entonces, un paradigma latinoamericano. En el momento actual, la justicia de ese sentimiento no es tan evidente.

Cerca de 1990, Costa Rica se ubicaba entre los países con mayor equidad en la educación. Una década más tarde pasa a una posición intermedia en la región y 20 años más tarde se ubica, junto al resto de los países centroamericanos en el grupo más desigual (Trejos, 2015, p. 6).

En 1990, Costa Rica era el cuarto país con mayor equidad en Latinoamérica. En el 2000, pasó a estar dentro del promedio. En 2010, solamente es mejor que los países centroamericanos. Eso muestra el menor avance relativo de Costa Rica en equidad educativa. El contraste más significativo es con Panamá, que ya se situaba en 1990 al mismo nivel de Costa Rica, mantuvo su posicionamiento relativo entre los cuatro países de mayor equidad educativa, en 20 años (Op. cit.). Actualmente, Panamá supera a Costa Rica.

Con todo y el considerable esfuerzo nacional en materia de oferta de educación superior; con 64 universidades, 5 públicas, 59 privadas, de las cuales 5 son internacionales y con una oferta de más de 1300 programas, el sistema de educación 
superior de Costa Rica tiene dos grandes flaquezas estructurales. Se trata de "la concentración de la presencia institucional en la zona urbana de la región Central y de la oferta académica en las áreas de Educación, Ciencias Sociales y Ciencias Económicas" (Op. cit., p. 248).

La dualidad del acceso a la educación superior se manifiesta en dos grandes fisuras: "las brechas de equidad por ingreso y por zona de procedencia" (Programa Estado de la Nación, 2017, p. 255). Asiste a la universidad solo un $11.2 \%$ de los jóvenes de 18 a 24 años de menor ingreso, contra el $58.1 \%$ de los de ingresos más altos. Es decir, "La participación de los jóvenes de hogares de mayores ingresos es más de cinco veces la de los jóvenes de hogares de menor ingreso" (Op. cit., p. 255).

La inequidad educativa por ingresos se repite y acentúa con las brechas territoriales. El 63.6\% de los jóvenes de 18 a 24 años provienen de la región central, pero esa población juvenil está sobrerrepresentada en la educación superior, con $76 \%$ de la matrícula. Mientras que el restante 36.4\% de los jóvenes están dispersos en los territorios periféricos, con muy baja y poco variada oferta educativa terciaria y componen solo el 24\% de la matrícula universitaria.

Los jóvenes de familias del quintil de menor ingreso son el $18.6 \%$ de la población juvenil, pero son solo el 7.4\% de la matrícula universitaria. Este y otros elementos de calidad, pertinencia, acceso y exclusión educativa, abordados en otros estudios, plantean la necesidad de acciones correctivas de las políticas educativas, para acortar brechas en modelo de desarrollo costarricense. El acceso a la universidad ocupa un lugar trascendental en estas acciones, porque en la sociedad del conocimiento, "los profesionales aventajan a la población de menor nivel educativo: tienen menor desempleo y subempleo, más altos ingresos y mayor formalidad en sus puestos de trabajo" (Programa Estado de la Nación, 2017, p. 243).
Por todo lo anteriormente expuesto, la promoción educativa debe considerarse, en sí misma, una política de convergencia. Pero cada institución de educación superior tiene desempeños que le son propios en la creación de capacidades, enriquecimiento del capital humano y mejoramiento de la competitividad de las empresas domésticas. Todo ello contribuye, desde variados ángulos, a adelantar procesos de convergencia tanto social y productiva, como territorial.

\section{EL DISEÑO ESPECÍFICO DE LA UNED PARA LA CONVERGENCIA EDUCATIVA}

No todas las instituciones educativas son igualmente idóneas para el objetivo de superar, de forma específica, las brechas de acceso a la educación terciaria. Ese fue, para la UNED, el objetivo determinado desde su misma concepción. La modalidad de educación a distancia de la UNED tiene el potencial de un impacto más efectivo en poblaciones excluidas. A pesar de su bajo presupuesto y de sufragar con esfuerzo propio un tercio de sus costos operativos, la UNED brinda una oferta particularmente atractiva para los segmentos sociales más urgidos de superación, donde la inequidad y la desigualdad golpean con más fuerza: territorios más alejados, estratos de menor ingreso, mujeres jefas de hogar.

Así lo consigna el Sexto Informe del Estado de la Educación (Programa Estado de la Nación, 2017). La UNED tiene solo el 6\% de la matrícula total, pero absorbe el $10.1 \%$ de la población estudiantil de las zonas rurales y el $15.4 \%$ del quintil de menor ingreso, mostrando una clara especialidad en esos segmentos donde se concentra, precisamente, el impacto más grave de la heterogeneidad estructural del país.

Cabe destacar diferentes contextos históricos entre las condiciones prevalecientes en el nacimiento de la UNED y las de la actualidad. Existía, entonces, "un ciclo virtuoso entre crecimiento económico y equidad social y en la 
construcción de una identificación colectiva con un proyecto nacional hegemónico" (Arias y Muñoz, 2007). Ese modelo entró en crisis y tuvo que ser sustituido. El nuevo modelo, sin embargo, nació y sigue inacabado.

La UNED fue creada entre dos mundos, entre el final de un modelo de desarrollo que aún no moría y el inicio de otro que aún no nacía. Dos elementos enmarcaron su singularidad. El primero es ser "una institución de educación superior especializada en enseñanza a través de los medios de comunicación social" (Ley 6044, art.1). El segundo, tener como objetivo primario y distintivo "Incorporar a la educación superior, con métodos idóneos y flexibles a quienes no hubieren podido incorporarse al sistema formal universitario" (Ley 6044, art. 2. c.).

Esas peculiaridades la definen como una universidad destinada a ofrecer educación a poblaciones con dificultades de acceso, la determinan a utilizar medios de comunicación social y le conceden el uso de flexibilidad académica. Cuando se creó la UNED, la prevaleciente homogeneidad productiva y la economía tradicional de mediados del siglo XX no habían convertido el acceso a la educación superior en una meta tan estratégica, como lo es ahora. En aquella sociedad, el desarrollo personal y colectivo no estaba tan fuertemente vinculado, al conocimiento como hoy.

La exclusión social tampoco acusaba los niveles de peligrosidad sociopolítica de esta época. La desigualdad de ingresos en Costa Rica, apenas uno de los componentes distintivos de la polarización social, ha ido aumentando sistemáticamente, en paralelo al desenvolvimiento del nuevo modelo. El Índice de Gini, que mide la desigualdad, en el que 0 valora la perfecta igualdad y 1, la total desigualdad, pasó de 0.38 en 1990 a 0.43 en 2001; luego, a 0.44 en 2009 y a 0.52 en 2016 (Arias, Sánchez, y Sánchez, 2009). Por eso, cuando nació la UNED, el rol estratégico que se le designó era diferente al que debería tener ahora. La heterogeneidad social y territorial que se vive hoy convierte el acceso de poblaciones excluidas en problema político y económico de primer orden.

Desde el punto de vista de los medios a su alcance, la creación de la UNED fue adelantada y prematura para sus tiempos. El pleno desarrollo tecnológico de facilitación de su modalidad a distancia llegaría después. La revolución tecnológica de las comunicaciones ofrece, a la modalidad a distancia, la posibilidad de optimizar su uso de "medios de comunicación social ", precisamente los que su ley de creación señala de su competencia especializada.

En estos tiempos, los "ganadores" o "perdedores" se definen también por el grado educativo alcanzado. En ese entorno, la UNED cobra toda su importancia, porque su facilitación de acceso a la educación superior para poblaciones excluidas es social y económicamente decisiva.

En resumen: en los tiempos actuales, las necesidades a las que responde la UNED son más agudas que nunca y los medios sociales de comunicación, de su especialidad, están en su momento histórico culminante. En contraste con el desarrollo de estas condiciones, la conciencia de la ampliación de su rol estratégico no ha madurado, como debería corresponder.

La fractura fundamental (...) ya no viene determinada únicamente por el acceso a la propiedad de los medios de producción, ni siquiera por el factor de división en grupos de estatus determinado por las diferencias en el consumo. El acceso a la información, y a través de ella al conocimiento, condiciona hoy en mayor medida la división y la estratificación social. (Barrenetxea y Cardona, 2002, p. 2).

De esa manera, aunque la UNED no fue originalmente concebida como una política pública de carácter estratégico, la evolución histórica de 
Costa Rica, transformaría ese rol. Esto cuando la población objeto de su atención y los medios específicos de su acción actualizaran su rol social, con la potencialidad de convertirla en instrumento privilegiado de superación de brechas.

Sin embargo, esta universidad no ha visto que se ponga al día una actualización de la importancia creciente de su rol. Por ello, en mucho sigue siendo visualizada como una universidad complementaria, en pugna con la creciente importancia de la modalidad de educación a distancia y de los instrumentos tecnológicos para implementarla. Ese desfase se manifiesta en los instrumentos de apoyo político y financiero que ha recibido, proporcionalmente invariables en el tiempo.

La relación de algunos perfiles sociodemográficos de las graduaciones de la UNED muestra su relevancia para superar brechas estructurales específicas que atiende con particular tino. Eso aboga, adicionalmente, a los argumentos anteriormente esgrimidos, en favor de la necesidad de actualizar su rol social.

Esta discusión, cobra particular relevancia, en estos momentos de crisis fiscal, cuando todo apunta a reducir gastos. Se analizarán algunos aspectos del perfil sociodemográfico de sus graduaciones para proponer exactamente la dirección opuesta, en el caso de la UNED.

\section{PERFILES SOCIODEMOGRÁFICOS DE GRADUACIONES DE LA UNED DE- MOSTRATIVOS DEL DESEMPEÑO DE SUS OBJETIVOS}

A pesar de contar con el más modesto presupuesto de las universidades públicas, la UNED puede mostrar, sin embargo, en algunos perfiles sociodemográficos de sus graduaciones, su desempeño para promover convergencia social. Para enfatizar esta contribución de la UNED a la superación de la heterogeneidad estructural, se escogieron tres características sociodemográficas de sus graduaciones:

- La proporción de estudiantes que provienen de estudios de secundaria a distancia.

- La proporción de graduados de zonas de IDS bajo y muy bajo.

- La proporción y características sociodemográficas de las mujeres graduadas en la UNED.

Se escogieron esos perfiles porque los obstáculos que más dificultan el acceso a la educación superior son, sobre todo:

- dificultad de acceso y baja calidad de la educación secundaria

- provenir de zonas de bajo índice de desarrollo

- vivir en zonas alejadas

- sumar a las condiciones anteriores, las desventajas propias de género: ser jefa de hogar, de bajo ingreso, con responsabilidades de cuido, desempleo, etc.

Servirá la exposición de esos perfiles como expresión del desempeño de la UNED como política pública estratégica en la promoción de procesos de convergencia social, económica, productiva y territorial, con sus consiguientes resultados en el mejoramiento de la cohesión social.

En lo que sigue, los datos estadísticos de las graduaciones 2013-2016 de la UNED se toman a partir de publicaciones del Centro de Investigación Estadística Institucional, bajo la autoría de Zamora y Gatgens (2017) y de Zamora y Charpentier (2018). 


\section{PRIMER PERFIL: ORIGEN EN SECUNDARIA A DISTANCIA DE GRADUADOS DE LA UNED}

Una brecha educativa de importancia estratégica para superar otras similares es la que existe entre los que completan la educación secundaria y los que no. Después de completar secundaria, se da otra brecha, entre los que entran a la universidad y los que no lo hacen.

En 2016, el 47.4\% de los jóvenes de Costa Rica entre 18 y 24 años no había completado secundaria (Programa Estado de la Nación, 2017). Esa diferencia es mucho mayor si se desagrega según nivel económico. En el quintil de mayores ingresos el 85\% logra completar segundaria. En el quintil de menores ingresos, en cambio, solo el 30.8\% lo hace. "La concentración de los quintiles superiores responde en parte a los patrones de fracaso en completar la educación secundaria, que es mayor en los estratos de menores ingresos" (Op. cit., p. 295).

En el acceso a la universidad el nivel económico provoca una brecha similar. Del segmento de menores ingresos entra a la universidad solo el $40 \%$ de los que completan secundaria, contra el $80 \%$ de los jóvenes del quintil de mayores ingresos (Op. cit., p. 255). La UNED comienza a combatir las dificultades de acceso ofreciendo, desde secundaria, su modalidad a distancia. Adicionalmente, al no requerir Prueba de Aptitud Académica (PAA), elimina un filtro de acceso, que se adiciona a los obstáculos socioeconómicos y territoriales. Eso explica, en parte, por qué su matrícula de primer ingreso de 5612 en 2009 es semejante a la de la UCR, 5817, en el mismo año (Op. cit.).

En un entorno de creciente desigualdad en la distribución de la riqueza y con un sistema de educación secundaria que está lejos de lograr la universalidad, no es previsible que, sin cambiar las reglas de ingreso a la educación superior, las universidades contribuyan a corregir las desigualdades de base. (Programa Estado de la Nación, 2011, p. 200).

El aspecto del perfil sociodemográfico analizado que se quiere destacar aquí, es la participación entre los graduados en la UNED 2013-2016 de $13 \%$ de varones y $15 \%$ de mujeres, que obtuvieron su bachillerato en la modalidad a distancia, modalidad que también ofrece la misma UNED. Este evento es especialmente ilustrativo si se toma en cuenta la movilidad interuniversitaria. No todos los jóvenes se gradúan en la universidad en la que originalmente se matricularon. En las otras universidades públicas, el 75\% de estudiantes que se gradúan lo hacen en la institución en la que se matricularon originalmente (Op. cit).

En la UNED no ocurre así. Solo el 21\% de los jóvenes que matriculan en la UNED logran graduarse en la UNED. El resto lo hace en otras universidades. Por eso, que el porcentaje de graduados de la UNED con origen en secundaria a distancia sea de $13 \%$ de varones y $15 \%$ de mujeres es un elemento destacado que muestra cómo esta universidad abre oportunidades, desde secundaria, a los segmentos sociales con difícil acceso a la educación. Muestra, también, cómo les sigue ofreciendo esa oportunidad hasta su graduación universitaria.

En esto conviene resaltar que, desde sus primeros años, la Universidad Estatal a Distancia entendió que su labor de atención a las personas excluidas de la enseñanza universitaria comenzaba con jóvenes y adultos que no habían terminado la educación media. En efecto, el más importante motivo de exclusión de los estudios superiores es la falta de conclusión de educación media. De esa preocupación nació la labor de la UNED en secundaria.

Mediante un convenio con el Ministerio de Educación Pública, se estableció el Colegio Nacional de Educación a Distancia (CONED), como modalidad de educación secundaria a 
distancia. El CONED está bajo la responsabilidad de la UNED y tiene como propósito la proyección de su metodología a la población excluida de la educación secundaria. Utilizando diferentes medios tecnológicos de información y comunicación, entrenando a los jóvenes en un aprendizaje autodirigido, autorregulado y autosuficiente, cuando estos estudiantes entran a los estudios superiores, en la misma UNED, han adquirido un alto nivel de disciplina y se han adaptado a la modalidad a distancia, lo que les facilita su continuidad y permanencia en el sistema y su éxito académico.

El CONED tiene 13 sedes distribuidas en todo el país y dedicadas a la educación secundaria a distancia. Entre 2008 y 2018, graduó a 2152 bachilleres y reporta 4499 graduados de III ciclo de la educación general básica (CONED, 2019). La labor de la UNED en secundaria es coronada con la responsabilidad que asumió de dirigir dos Colegios Cientíicos adscritos a ella, uno en Limón y otro en Alajuela. Ambos ganaron el Premio Nacional de la Excelencia Académica. El de Limón, en 2014 y el de Alajuela, en 2016. Ambos por haber obtenido el mejor promedio en las seis pruebas nacionales de Bachillerato.

\section{SEGUNDO PERFIL: SOBRERREPRESENTACIÓN DE IDS BAJO Y MUY BAJO ENTRE LOS GRADUA- DOS DE LA UNED}

"La distribución de recintos de la UNED cubre de manera similar todas las regiones" (Programa Estado de la Nación, 2017, p. 248); ese es el punto de partida para entender su impacto en zonas de menor desarrollo relativo. Debe entenderse que las brechas territoriales engloban y enmarcan todas las fisuras sociales: desigualdades de ingreso, brechas educativas, menores oportunidades de empleo, disparidad de servicios, patrones menores de consumo, pocas iniciativas de emprendimiento y, también, dificultad de acceso a la universidad. De ahí, el gran significado que tiene el perfil territorial de las graduaciones de la UNED, con las poblaciones específicas a las que atiende en las regiones.

La relación de concentración-dispersión de los habitantes expresa estos contrastes, resultado de la dualidad económica. Las poblaciones tienden a congregarse en las zonas de mayor dinamismo y a abandonar las zonas deprimidas. En el 16.7\% del territorio se aglutina el $62.2 \%$ de la población (Figura 1). En el $83.3 \%$ del territorio vive el $37.8 \%$ de la población restante, de forma generalmente

FIGURA 1

\section{DISTRIBUCIÓN PORCENTUAL DE POBLACIÓN REGIÓN CENTRAL Y REGIONES PERIFÉRICAS*}

\section{- Región Central $\quad$ - Regiones Periféricas}

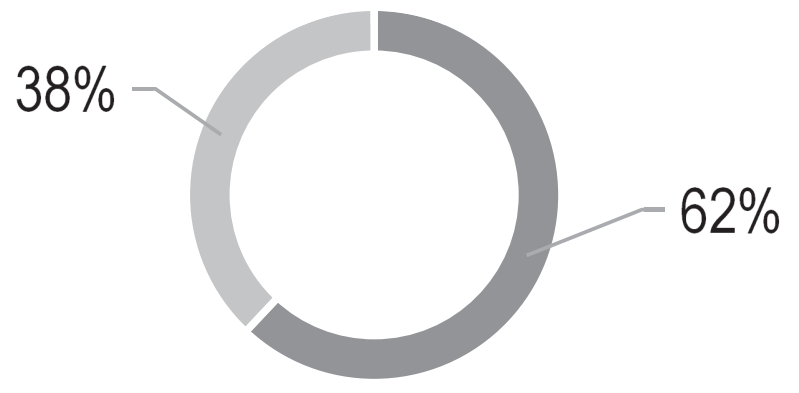

Fuente: Elaboración a partir de MIDEPLAN (2016)

*Nota: Región Chorotega (8\%), Región Huetar Norte (8\%), Región Huetar Atlántica (9\%), Región Brunca (7\%), Región Pacífico Central (6\%) 
dispersa, con menores ingresos, mayor desempleo y escasos sistemas de soporte social y económico (MIDEPLAN, 2017, p. 43).

"Las brechas de equidad por ingreso y por zona de procedencia son una característica estructural de la educación universitaria" (Programa Estado de la Nación, 2017, p. 255). Los hogares de mayor ingreso tienen cinco veces más oportunidades de tener hijos en la universidad que los de menor ingreso. Entre los jóvenes que terminan secundaria que provienen de hogares de menor ingreso, solo el 40\% entra a la universidad, contra el $80 \%$ que desciende de hogares más ricos (Op. cit.). Bajos niveles de ingreso obstaculizan dedicarse exclusivamente al estudio y la necesidad de trabajar es un filtro adicional de la modalidad presencial, que no existe en la modalidad a distancia.

A ese obstáculo económico se suman múltiples dimensiones de exclusión de los territorios de bajo nivel de desarrollo relativo. Un análisis de las graduaciones por regiones, según su Índice de Desarrollo Social (IDS), considera la superación de barreras de acceso que van más allá de la dimensión económica. Esos "otros" componentes, agrupados en el IDS, brindan una perspectiva más sistémica de los obstáculos de acceso a la educación superior. El IDS bajo y muy bajo incluye, además, el entorno más amplio de elementos que dificultan procesos de aprendizaje, tales como el rezago de educación en las familias, factor coadyuvante que demarca las posibilidades de completar la educación secundaria, lo que, a su vez, dificulta el acceso a la universidad.

Para ilustrar este punto, en 2010, un promedio del $8 \%$ de las familias tenía estudiantes en las universidades públicas. Pero si los jefes de hogar no tenían primaria, el porcentaje de participación universitaria bajaba hasta el 2\%. En cambio, si los padres tenían secundaria completa, la posibilidad de acceso a la universidad subía hasta el 12\%. Las mejores posibilidades de acceso universitario las tenían los jóvenes de familias con educación universitaria. Hasta el 16\% de estas familias tenía hijos en la universidad (Programa Estado de la Nación, 2013).

En las graduaciones de la UNED 2013-2016, existe una sobrerrepresentación de estudiantes que provienen de zonas de IDS bajo y muy bajo. Es decir, su proporción de participación entre los graduados es mayor que la proporción de población de zonas con esos IDS.

Si se considera la residencia de las personas, según IDS, el 30.55\% de la población del país se encuentra en distritos de IDS bajo o muy bajo y el $69.47 \%$ en distritos de desarrollo medio o alto. Se confirma, así, la alta concentración en zonas de mayor dinamismo económico y social. En contraste con esto, la proporción de graduados de la UNED, por origen según IDS bajo o muy bajo de sus distritos de residencia, es en todos sus grados académicos mucho mayor que su proporción poblacional.

En un análisis estadístico del perfil sociodemográfico de las graduaciones de la UNED, Zamora y Charpentier (2017) constatan, de manera muy característica, una mayor participación de graduados de zonas de menor índice de desarrollo, especialmente mujeres (ver figuras 2 y 3). Tienen origen en distritos de bajo o muy bajo IDS el $45.9 \%$ de sus diplomados, el $54.3 \%$ de sus profesorados, el $42.2 \%$ de sus bachilleratos y el $37.9 \%$ de sus licenciaturas;

el $73.0 \%$ de las personas graduadas viven en cantones de Menor Desarrollo Relativo y, de forma más específica 44 de cada 100 personas graduadas residen en cantones de nivel bajo y muy bajo. Se observa que 74 de cada 100 mujeres graduadas vive en cantones de Menor Desarrollo relativo (Zamora y Charpentier, 2017, p. 17).

Se muestra un contraste. La proporción de graduados con origen de IDS bajo o muy bajo es 


\title{
DISTRIBUCIÓN DE PERSONAS SEGÚN IDS 2013 DEL CANTÓN DE RESIDENCIA
}

\section{- Mayor y medio IDS - Bajos y muy bajos IDS}

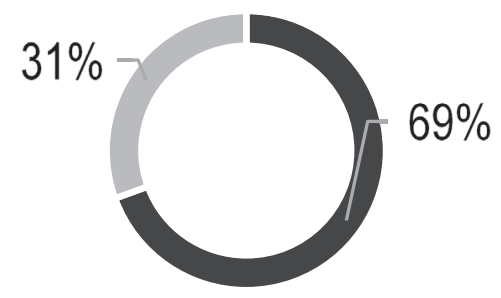

Fuente: Elaboración propia a partir de MIDEPLAN (2017)

\author{
FIGURA 3 \\ DISTRIBUCIÓN DE GRADUADOS SEGÚN IDS 2013 DEL CANTÓN DE RESIDENCIA
}

- Mayor Desarrollo Relativo - Menor Desarrollo Relativo

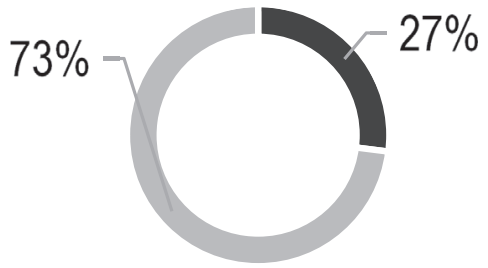

Fuente: Elaboración propia a partir de Base de datos del cuestionario de personas graduadas 2013-2016. CIEI-UNED.

mayor que la proporción de graduados con origen de IDS alto o medio. Eso se acentúa aún más, si se ponen esas proporciones en correspondencia con los pesos poblacionales de su origen por IDS. Todo ello muestra en los perfiles de las graduaciones de la UNED 2013-2016 un fuerte impacto como política afirmativa que favorece preferentemente a las poblaciones excluidas. Aquí resalta la huella convergente de la fuerza estratégica que tiene su modalidad educativa a distancia, porque potencia el desarrollo humano regional en las zonas más deprimidas y facilita la superación de brechas territoriales.

\section{TERCER PERFIL: MUJERES ENTRE LOS GRADUA- DOS DE LA UNED}

En Costa Rica se ha venido dando un incremento de la matrícula y las graduaciones de mujeres en todas las universidades, superando, en algunas ocasiones, las de los varones. La UNED, sin embargo, lo ha hecho con mucha mayor fuerza, alcanzando una matrícula del $63.83 \%$ de mujeres en 2017 (Figueroa y Gatgens, 2018). Un análisis historiográfico muestra la prevalencia de las mujeres en la UNED, tanto en la matrícula de primer ingreso de pregrado, como de posgrado (ver figuras 4 y 5 ). 


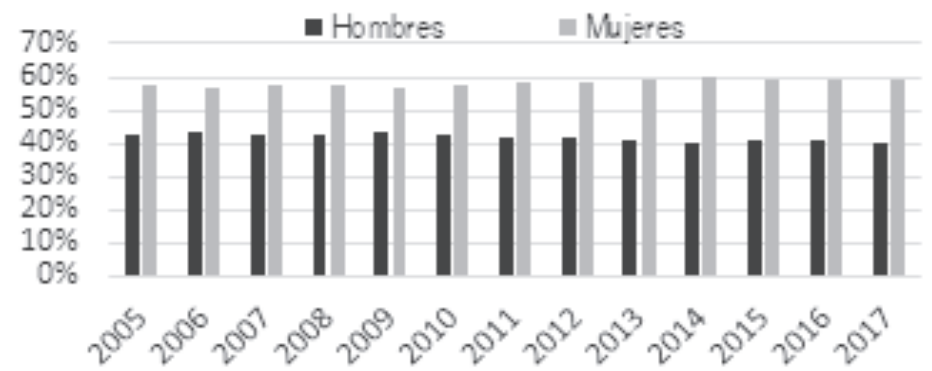

Fuente: Elaboración propia a partir de Anuario estadístico 2017. (IEI-UNED. 2018.

\section{FIGURA 5 \\ MATRÍCULA ANUAL DE ESTUDIANTES DE PRIMER INGRESO EN POSGRADO SEGÚN SEX0. 2005-2017}

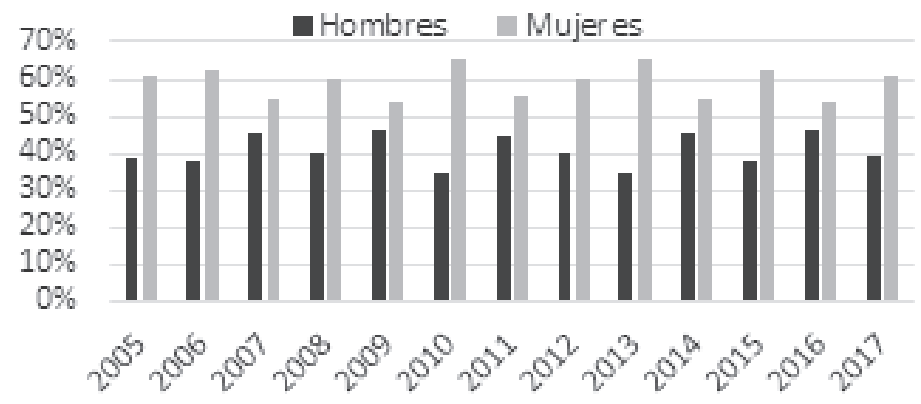

Fuente: Elaboración propia a partir de Anuario estadístico 2017. (IEI-UNED. 2018.

Más notable aún es el éxito académico de las mujeres que estudian en la UNED. La proporción de graduación de mujeres excede la proporción de mujeres graduadas en otras universidades. En la UNED, la graduación de mujeres entre 2013 y 2017 alcanzó un promedio de $75.2 \%$ del total de graduados (Zamora y Charpentier, 2017). Es decir, tres de cada cuatro personas graduadas de la UNED son mujeres.

Lo anterior se traduce en que las mujeres encuentran en la UNED un entorno más acoplado a sus necesidades, porque desertan o cambian de universidad menos que los hombres. Este dato explica su proporción diferente entre matrícula y graduación; mientras que las mujeres representan el 60\% en la matrícula son, en cambio, el 75\% en las graduaciones. Los hombres, que alcanzan un 40\% en la matrícula, en las graduaciones ven reducida su representación al 25\% (Ver figura 6).

Sin embargo, ese resultado tiene una relación directa con las políticas universitarias de la UNED, de plena conformidad con sus objetivos institucionales, lo cual se refleja en la proporción de becas académicas asignadas a las mujeres en comparación a los hombres. Analizando los datos del Anuario Estadístico 2017, del CIEl de la 
FIGURA 6

CONTRASTE DE LA PROPORCIÓN ENTRE MATRÍCULA Y GRADUACIONES SEGÚN SEX0. 2013-2017

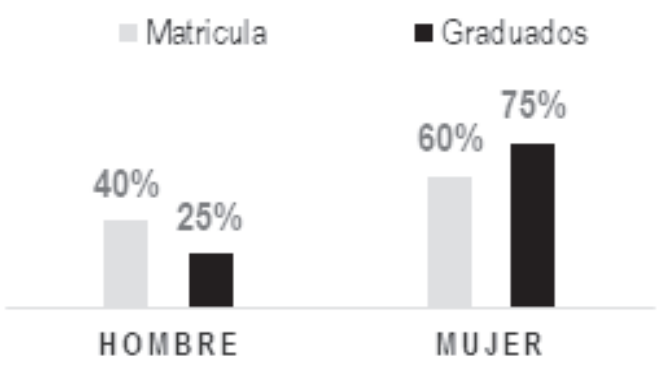

Fuente: Elaboración propia a partir de Anuario estadístico 2017 y perfil de las personas graduadas. 2013-2017. (IEI-UNED. 2018.

FIGURA 7

BECAS ASIGNADAS POR SEX0. 2008-2017

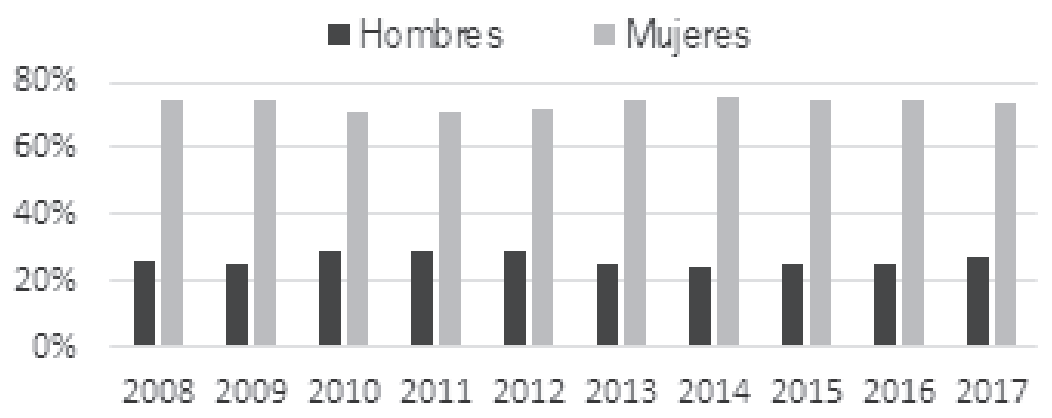

Fuente: Elaboración propia a partir de Anuario estadístico 2017. (IEI-UNED. 2018.

UNED (Figueroa y Gatgens, 2018), la UNED asignó, entre 2008 y 2017, un promedio de 74\% de sus becas a las mujeres; los hombres recibieron solamente el $26 \%$ de las becas estudiantiles. Curiosa esa relación de proporcionalidad entre becas y graduaciones.

La serie historiográfica de la asignación de becas de la UNED muestra, en la práctica, un rasgo constante de política afirmativa de género, aunque no exista dicha decisión como norma institucional (ver figura 7). Es preciso notar que la mayor proporción de mujeres graduadas (51.6\%) lograron estudiar por medio de becas otorgadas por la UNED. "Por su parte los hombres se financian mayormente con fondos generados por ellos mismos (77.3\%)" (Zamora y Charpentier, 2018, p. 19).

A pesar del significado que implica, como el empoderamiento profesional de las mujeres, la proporción de mujeres matriculadas, becadas y graduadas no lo dice todo. Cuando se trata de este sector de la población, la situación familiar se suma a los demás elementos de su perfil sociodemográfico, como obstáculo suplementario para alcanzar la educación superior. Entre las mujeres graduadas de la UNED, más que en otras 
universidades, es muy grande la proporción que no están casadas ni en unión libre y que son madres solteras, en muchas ocasiones jefas de hogar que además trabajan.

En las graduaciones de la UNED se encuentra un perfil característico de mujeres que enfrentan adversidades que exacerban las propiamente vinculadas con desventajas asociadas al género. Las mujeres graduadas completan algunas o todas las características de un perfil que describe el universo acumulado de las desventajas de género que, de forma realmente sistémica, encuentran salida educativa de nivel superior, en la UNED.

Las graduaciones de mujeres las muestran jóvenes, entre 21 y 30 , en un $49.5 \%$ años; de muy bajo ingreso por provenir el $16.2 \%$ de zonas de bajo y el $28.2 \%$ de muy bajo desarrollo; $46.9 \%$ son solteras; muchas de ellas madres, jefas de hogar. Además, 34.4\% de las mujeres graduadas trabajan y estudian.

Adicionalmente y para mejor precisar el significado que tiene la graduación en la UNED, "del total de las personas que están laborando al momento de graduarse, más del 50\% señala que dicho trabajo y la carrera en la que se van a titular tienen un grado total de relación" (Zamora, 2017, p. 29). Eso ofrece una mejor visualización de la riqueza que significó para todas esas mujeres haber tenido acceso a una opción educativa superior a distancia.

\section{CONCLUSIONES}

La Universidad Estatal a Distancia fue fundada en 1977, en una sociedad que tenía una cohesión y homogeneidad social mayor que la actual. Su ley creadora le asignó como objetivo incorporar a los estudios universitarios a quienes no habían podido hacerlo, a través de medios de comunicación social.

Desde entonces, y con la internacionalización de Costa Rica en la globalización, se han producido cambios estructurales, caracterizados por dualidades productivas y fuerte heterogeneidad en todos los órdenes de la vida común. La desigualdad de ingresos y las diferencias contrastantes entre zonas con índices sociales de desarrollo han provocado pérdida de cohesión social. Crece la consciencia del carácter inconcluso del modelo de desarrollo costarricense.

La creación de la UNED fue una política educativa previsora, pero no en la medida de prioridad que exigen las necesidades actuales. La heterogeneidad del país, derivada de la internacionalización de Costa Rica, la transformación mundial de la importancia de la educación y la revolución tecnológica de la información y las comunicaciones, con la Sociedad del Conocimiento, son elementos que abogan por un debate más amplio del rol social y del peso estratégico de la UNED.

Como lo consignan diversos autores, citados supra, existe cada vez más consciencia de la necesidad de diseñar e implementar políticas de convergencia para acortar los extremos de esta polarización. Se demandan, entre otras, políticas productivas internas, políticas sociales y educativas eficientes y desarrollo institucional más homogéneo.

De forma paralela al crecimiento de la heterogeneidad nacional, en el orden mundial se ha producido una disruptiva revolución en las tecnologías de la información y la comunicación, suscitando lo que se ha dado en llamar la Sociedad del Conocimiento. Estos cambios tecnológicos introdujeron también nuevas brechas, lo cual hizo que el acceso a las universidades desarrollara mayor importancia que cuando nació la UNED. Pero también que su modalidad a distancia cuenta con instrumentos tecnológicos más decisivos.

Al tiempo que, para el progreso humano, se hizo más decisivo que en el pasado el acceso a la universidad de poblaciones excluidas y que se han revolucionado los medios sociales para facilitar dicho acceso, desde 1977 no ha cambiado, la visión de rol estratégico de la UNED, desde la 
perspectiva de los diseñadores de políticas públicas. Eso es así, al menos, en la medida en que lo estático de las prioridades se expresa en la permanente e inmutable proporcionalidad relativa de sus asignaciones presupuestarias.

Al analizar tres características sociodemográficas de recientes graduaciones de la UNED, aparece: (1) que una proporción significativa del origen educativo de sus graduados es de jóvenes con bachillerato en la modalidad a distancia; es decir, excluidos de la educación formal en institutos regulares de segunda enseñanza y rescatados al sistema educativo por la UNED; (2) que la proporción de sus graduados de zonas de IDS bajo y muy bajo es superior a la distribución poblacional de esas zonas, mostrando el mayor impacto de su modalidad educativa en esos segmentos de población y (3) que las características sociodemográficas de las graduaciones de las mujeres, así como la proporción de su participación muestran la capacidad que tiene la UNED para enfrentar las desventajas de género.

En esas tres características se destaca la fuerza de convergencia de la UNED para equilibrar las asimetrías heterogéneas de Costa Rica. Esta fuerza sería potencializada con un reforzamiento del rol social de la UNED, como política estratégica. Como se deduce de esta exposición, quedó ilustrado el respaldo investigativo de literatura de referencia y de estadística relevante de su hipótesis de partida y de su propuesta de tesis central.

Según se describió, a pesar de la gran inversión social y educativa de Costa Rica, el Estado no ha contrarrestado suficientemente los efectos negativos de la globalización. Eso ha dado lugar a la creación de condiciones duales de variada naturaleza en muchos órdenes de la vida común, menoscabando la cohesión social, condición de la más alta gravedad. Esta degradación de la cohesión necesita enfrentarse con políticas públicas estratégicas de restitución, especialmente las educativas, ya que la educación es un componente decisivo de movilidad social y, en ese sentido, de convergencia entre las brechas socioeconómicas creadas.

De ahí que el análisis de literatura de referencia y el estudio de estadística relevante permite responder positivamente a las preguntas metodológicas inicialmente diseñadas en el planteamiento del problema. Efectivamente, dentro de las limitaciones de su presupuesto, (a) la UNED responde su objetivo fundacional, ya que (b) en las características sociodemográficas expuestas de sus graduaciones, resalta el impacto potencial que tiene la UNED como política de inclusión restaurativa de la cohesión social, con sus contribuciones educativas a la superación de las brechas socioeconómicas, territoriales y de género existentes. Eso (c) muestra la particular pertinencia de la UNED como política pública de convergencia.

En esta misma línea de pensamiento, se recomendarían futuras investigaciones estadísticas y de campo para abordar esta temática con análisis y cuestionarios específicos recabados de fuentes primarias, abordando cada uno de estos perfiles. Sería, por ejemplo, recomendable un estudio sociodemográfico de las mujeres graduadas de la UNED, desde secundaria hasta su situación laboral actual.

En resumen, se ofrecen los perfiles estadísticos institucionales de esas graduaciones para validar, al menos parcialmente, la modalidad educativa a distancia, tal como es emprendida y promovida por la UNED. Esto es, como una política pública que ameritaría estudios más amplios de revalorización dentro de una estrategia-país más holística para restaurar la cohesión social perdida.

\section{REFERENCIAS}

Arias, R. \& Muñoz, J. J. (2007). Reforma Económica y Modelo de Promoción de Exportaciones: logros y vacíos de la política de desarrollo de las últimas dos décadas. Revista de Ciencias Económicas, 25(1), 15-40. Recuperado de https://re- 
vistas.ucr.ac.cr/index.php/economicas/article/ view/7174/6854

Arias, R., Sánchez, R., \& Sánchez, L. (2011). Transformación productiva y desigualdad en Costa Rica. Revista de Ciencias Económicas, 29(1), 59-95. Recuperado el 12-08-2018 de https://revistas. ucr.ac.cr/index.php/economicas/article/download/7035/6720

Asamblea Legislativa. Ley No 6044. (1977). Ley de Creación de la Universidad Estatal a Distancia (UNED). San José, Costa Rica: "La Gaceta" No.50 del 12 de Marzo de 1977. Recuperado de https:// www.uned.ac.cr/academica/images/Normativa/ Ley_de_creacion.pdf

Barrenetxea, M. \& Cardona, A. (2002). La brecha digital como fuente de nuevas desigualdades en el mercado de trabajo. Bilbao, España: Universidad del País Vasco. Recuperado de http://webs.ucm.es/info/ ec/jec8/Datos/documentos/comunicaciones/ Laboral/Barrenetxea\%20Miren.PDF

Beverinotti, J., Coj-Sam, J., \& Solís, G. (2015). Dualidad productiva y espacio de crecimiento para las pymes en Costa Rica. San José, Costa Rica: Banco Interamericano de Desarrollo.

Comisión Económica para América Latina y el Caribe (CEPAL). (2011). El desarrollo inclusivo en América Latina y el Caribe: ensayos sobre políticas de convergencia productiva para la igualdad. Santiago, Chile: CEPAL. Recuperado de https://www.cepal. org/es/publicaciones/2594-desarrollo-inclusivo-america-latina-caribe-ensayos-politicas-convergencia

Colegio Nacional de Educación a Distancia (CONED). (2019). Estadísticas a nivel nacional. San José, Costa Rica: Sitio Web del Colegio Nacional de Educación a Distancia de la UNED. Recuperado de https://coned.uned.ac.cr/vida-estudiantil/estadisticas-a-nivel-nacional

Figueroa, L. \& Gatgens, D. (2018). Anuario estadístico 2017. San José, Costa Rica: Centro de Investigación y Evaluación Institucional (CIEI) de la UNED. Recuperado de https://www.uned.ac.cr/viplan/ images/ciei/ANUARIO_2017/Anuario_2017_versi\%C3\%B3n_final_19-05-18.pdf
Govaere, V. \& Ruiz, H. (2013). Heterogeneidad y Desigualdad en el modelo costarricense: las mejores tres prácticas latinoamericanas de fomento fiscal a la inversión privada en I+D+i. Revista Nacional de Administración, 4(1), 7-26.

Govaere, V. (2018a). Prolegómenos de impactos y procesos de los TLC en Costa Rica. San José, Costa Rica: EUNED (pendiente de publicación).

Govaere, V. (2018b). Un voto defensivo. San José, Costa Rica: Periódico La Nación, 20 de febrero. Recuperado de https://www.nacion.com/opinion/ columnistas/un-voto-defensivo/SBHEA6ABKJFFHFNZQ7O3U74GBY/story/

Jarauta, F. (1998). Escenarios de La Globalización. Rosario, Argentina: Homo Sapiens Ediciones.

Ministerio de Planificación Nacional y Política Económica (MIDEPLAN). (2017). Indice de Desarrollo Social 2017. San José, Costa Rica: MIDEPLAN Recuperado de http://www.conicit.go.cr/biblioteca/ publicaciones/publica_cyt/informes/Indice_Desarrollo_Social_2017.pdf

Ocampo, J. (2002). Globalización y desarrollo social. Santiago, Chile: Segundo Encuentro de ex-Presidentes Latinoamericanos. Recuperado de https:// www.cepal.org/prensa/noticias/comunicados/2/9812/presentacionjao.pdf

Ocampo, J. A. \& Martin, J. (2003). Globalization and Development: A Latin American and Caribbean Perspective. Stanford, EE.UU.: Stanford University Press.

Ottone, E. \& Sojo, A. (2007). Cohesión social: inclusión y sentido de pertenencia en América Latina y el Caribe. Santiago, Chile: CEPAL.

Pérez, H. (2004). Educación, capital humano y movilidad social en Costa Rica. Un primer análisis de los datos del censo de 2000. San José, Costa Rica: Centro Centroamericano de Población (CCP) de la Universidad de Costa Rica. Recuperado de https://ccp.ucr.ac.cr/bvp/pdf/censo2000/libro-censo/3.1-Perez-2.doc.pdf

Programa Estado de la Nación. (2008). XV informe. Capítulo 6: Clases sociales. San José, Costa Rica: Estado de la Nación. Recuperado de https://www. 
estadonacion.or.cr/files/biblioteca_virtual/015/ XV_informe_capitulo_6.pdf

Programa Estado de la Nación. (2011). Tercer Informe Estado de la Educación. San José, Costa Rica: Estado de la Nación. Recuperado de https:// www.estadonacion.or.cr/97-estado-de-la-educacion/166-informe-iii-estado-de-la-educacion

Programa Estado de la Nación. (2013). Cuarto informe Estado de la Educación. Programa Estado de la Nación. San José, Costa Rica: Estado de la Nación. Recuperado de https://www.estadonacion.or.cr/files/biblioteca_virtual/educacion/004/9-Cap-4.pdf

Programa Estado de la Nación. (2017). Sexto Informe Estado de la Educación. Programa Estado de la Nación. San José, Costa Rica: Estado de la Nación. Recuperado de https://www.estadonacion.or.cr/ educacion2017/informe-para-descarga.html

Roubini, N. (2016). Las líneas políticas divisorias de la globalización. New York, EE.UU: New York Times. Recuperado de http://www.nacion.com/opinion/foros/lineas-politicas-divisorias-globalizacion_0_1571642827.html

Salazar-Xirinach, J. M., Nübler, I., \& Kozul-Wriht, R. (2014). Transformando las economías: Haciendo que la política industrial funcione para el crecimiento, el empleo y el desarrollo. Ginebra, Suiza: Organización Internacional del Trabajo.

Serrano, C. (2005). La política social en la globalización: programas de protección en América Latina. New York, EE.UU.: United Nations Publications.

Stiglitz, J. (2010). El malestar de la globalización. Madrid, España: Penguin Random House Grupo Editorial.

Stiglitz, J. (2015). La gran brecha: Qué hacer con las sociedades desiguales. Madrid, España: Penguin Random House Grupo Editorial.

Trejos, J. D. (2015). Quinto informe del Estado de la Educación. La desigualdad en la distribución de la educación en Costa Rica: una mirada comparativa de los últimos 25 años. San José, Costa Rica: Estado de la Nación.
Zamora, T., \& .Charpentier, E. (2018). Perfil de las personas graduadas 2013-2017. Documento CIEI-0262018. San José. Costa Rica: Centro de Investigación y Evaluación Institucional (CIEI) de la UNED. Recuperado de https://www.uned.ac.cr/viplan/ images/ciei/INVESTIGACIONES_2018/26_Informe_de_Perfil_de_personas_graduadas_de_la_ UNED_2013-2017.pdf

Zamora, T. (2017). Informe de las personas graduadas 2013-2016. Documento CIEI 013-2017. San José, Costa Rica: Centro de Investigación y Evaluación Institucional de la UNED. Recuperado de https:// www.uned.ac.cr/viplan/images/ciei/INVESTIGACIONES_2017/Informe_de_personas_graduadas_2013-2016.pdf

Recibido:13 de agosto de 2018

Aceptado:05 de marzo de 2019 